\title{
Evidence of a nodal line in the superconducting gap symmetry of noncentrosymmetric $\mathrm{ThCoC}_{2}$
}

\author{
A. Bhattacharyya, D. T. Adroja, K. Panda, Surabhi Saha, \\ Tanmoy Das, A. J. S. Machado, O. V. Cigarroa, \\ T. W. Grant, Z. Fisk, A. D. Hillier and P. Manfrinetti
}

\section{Published version information}

Citation: A Bhattacharyya et al. "Evidence of a nodal line in the superconducting gap symmetry of noncentrosymmetric ThCoC 2 ." Physical Review Letters, vol. 122, no. 14 (2019): 147001.

DOI: $10.1103 /$ PhysRevLett.122.147001

This version is made available in accordance with publisher policies. Please cite only the published version using the reference above. This is the citation assigned by the publisher at the time of issuing the APV. Please check the publisher's website for any updates. 


\title{
Evidence of a Nodal Line in the Superconducting Gap Symmetry of Noncentrosymmetric $\mathrm{ThCoC}_{2}$
}

\author{
A. Bhattacharyya, ${ }^{1,2,3,{ }^{*}}$ D. T. Adroja ${ }^{2,3, \dagger}$ K. Panda, ${ }^{1}$ Surabhi Saha, ${ }^{4}$ Tanmoy Das, ${ }^{4, \$}$ A. J. S. Machado, ${ }^{5}$ \\ O. V. Cigarroa, ${ }^{5,6}$ T. W. Grant, ${ }^{5,6}$ Z. Fisk, ${ }^{6}$ A. D. Hillier, ${ }^{2}$ and P. Manfrinetti ${ }^{7}$ \\ ${ }^{1}$ Department of Physics, Ramakrishna Mission Vivekananda Educational and Research Institute, \\ Belur Math, Howrah 711202, West Bengal, India \\ ${ }^{2}$ ISIS Facility, Rutherford Appleton Laboratory, Chilton, Didcot Oxon, OX11 OQX, United Kingdom \\ ${ }^{3}$ Highly Correlated Matter Research Group, Physics Department, University of Johannesburg, \\ P. O. Box 524, Auckland Park 2006, South Africa \\ ${ }^{4}$ Department of Physics, Indian Institute of Science, Bangalore 560012, India \\ ${ }^{5}$ Escola de Engenharia de Lorena, Universidade de São Paulo, P. O. Box 116, Lorena, São Paulo, 12602810 \\ ${ }^{6}$ Department of Physics and Astronomy, University of California-Irvine, Irvine, California 92697, USA \\ ${ }^{7}$ Department of Chemistry, University of Genova, 16146 Genova, Italy
}

(Received 14 October 2018; revised manuscript received 14 January 2019; published 10 April 2019)

\begin{abstract}
The newly discovered noncentrosymmetric superconductor $\mathrm{ThCoC}_{2}$ exhibits numerous types of unconventional behavior in the field dependent heat capacity data. Here we present the first measurement of the gap symmetry of $\mathrm{ThCoC}_{2}$ by muon spin rotation and relaxation $(\mu \mathrm{SR})$ measurements. The temperature dependence of the magnetic penetration depth measured using the transverse field $\mu \mathrm{SR}$ experiment reveals the evidence of a nodal pairing symmetry. To understand this finding, we carry out calculations of the superconducting pairing eigenvalue and eigenfunction (pairing symmetry) due to the spin-fluctuation mechanism by directly implementing the $a b$ initio band structures. We find that the system possesses a single Fermi surface with considerable three dimensionality and a strong nesting along the $k_{z}$ direction. Such nesting promotes a superconducting state with a $\cos k_{z}$-like pairing symmetry with a prominent nodal line on the $k_{z}= \pm \pi / 2$ plane. The result agrees well with the experimental data.
\end{abstract}

DOI: 10.1103/PhysRevLett.122.147001

In recent years, noncentrosymmetric (NCS) superconductors have attracted considerable attention in condensed matter physics, both theoretically and experimentally [1,2]. The loss of inversion symmetry in these NCS compounds generates an asymmetric electrical field gradient in the crystal lattice, and, thereby, produces a Rashba-type spinorbit coupling (RSOC, energy scale $\sim 10-100 \mathrm{meV}$ ) [3-6]. In the presence of RSOC, the spin of the Cooper pair is not a good quantum number; the spin degeneracy of the conduction band is lifted except at time-reversal invariant momenta, and hence it allows for an admixture of spinsinglet and spin-triplet states in Cooper pairs formation $[4,5]$. If the triplet part is large [7], the superconducting gap reveals line or point nodes in the order parameter. The discovery of unconventional superconductivity (USC) in $\mathrm{CePt}_{3} \mathrm{Si}$, with $T_{c}=0.75 \mathrm{~K}$, has spurred theoretical and experimental works to find out the effect of the lack of inversion symmetry on NCS. [8-10]. USC in applied pressure was subsequently observed in NCS heavy fermion (HF) compounds [11-13], such as $\operatorname{CeTSi}_{3}(T=\mathrm{Rh}, \mathrm{Ir})$ [14-16] and $\mathrm{CeTGe}_{3}(T=\mathrm{Co}, \mathrm{Rh}$, and Ir) [17]. To date, USC properties, e.g., a nodal gap structure and/or a large upper critical field, have been presented in a few weakly correlated NCS superconductors, including $\mathrm{Li}_{2}\left(\mathrm{Pd}_{1-x} \mathrm{Pt}_{x}\right)_{3} \mathrm{~B}$
[18,19], $\mathrm{Y}_{2} \mathrm{C}_{3}$ [20], $\mathrm{Mo}_{3} \mathrm{Al}_{2} \mathrm{C}$ [21], $\mathrm{Mg}_{10} \mathrm{Ir}_{19} \mathrm{~B}_{16}$ [22], and with special attention to $\mathrm{LaNiC}_{2}$ [23].

$\mathrm{ThCoC}_{2}$ with $T_{c}=2.3 \mathrm{~K}$, crystallizes in the NCS $\mathrm{CeNiC}_{2}$-type orthorhombic structure with space group Amm 2 (No. 38), in which a mirror plane is missing along the $c$ axis [24-26], as displayed in Fig. 1(a). The upper critical field $\left(H_{c 2}\right)$ vs temperature phase diagram confirms a positive curvature [25], and the electronic specific heat coefficient at a low temperature exhibits a square root field dependence $\gamma(H) \propto \sqrt{H}$, which indicates the presence of nodes in the superconducting order parameter [27-30] and a potential USC state in $\mathrm{ThCoC}_{2}$. The $\gamma(H) \propto \sqrt{H}$ property is often considered to be a generic signature of the presence of $d$-band and HF superconductors [28-30]. Moreover, examples of positive curvature in a $H_{c 2}(T)$ phase diagram include $\mathrm{LnNi}_{2} \mathrm{~B}_{2} \mathrm{C}(\mathrm{Ln}=\mathrm{Lu}$ and $\mathrm{Y})$ [31,32], $\mathrm{MgB}_{2}$ [33], the $\mathrm{NCS} \mathrm{Li}_{2}(\mathrm{Pd}, \mathrm{Pt})_{3} \mathrm{~B}$ [34], and the NCS HF CeRhSi [35]. The isostructural compound $\mathrm{LaNiC}_{2}$ displays USC below $T_{c}=2.7 \mathrm{~K}$ with broken time-reversal symmetry (TRS) as observed by $\mu$ SR measurements, a nodal energy gap as observed by very-low-temperature magnetic penetration depth measurements, and possible multigap superconductivity due to the modest value of the RSOC $[23,36]$. Motivated by these results, in this Letter, we investigate the 

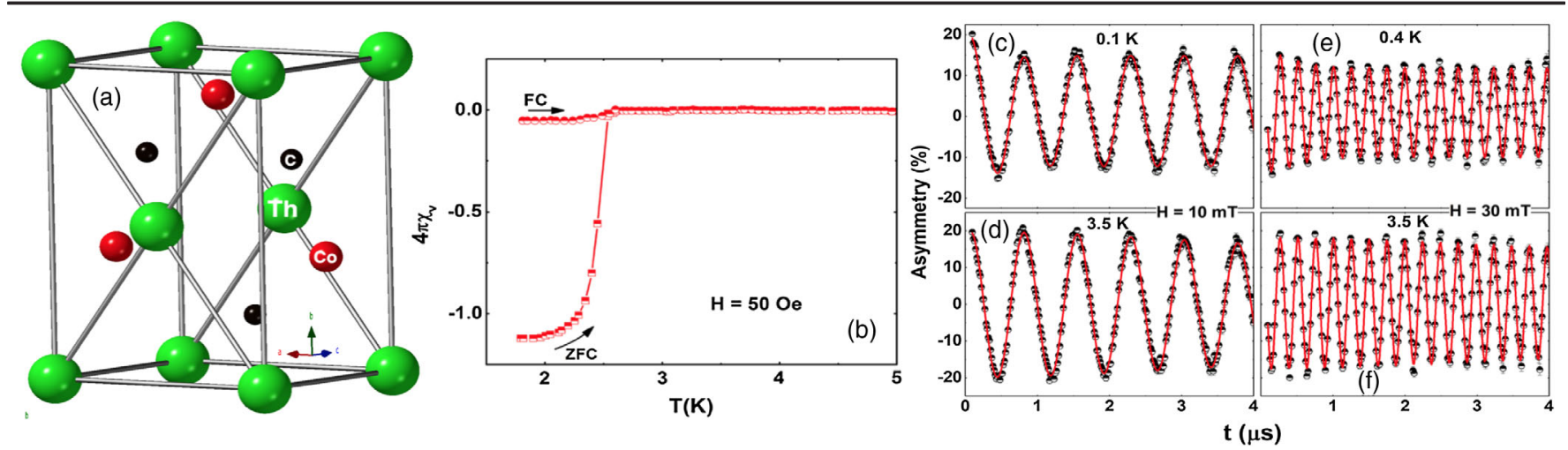

FIG. 1. (a) The orthorhombic crystal structure of $\mathrm{ThCoC}_{2}$, where green, red, and black symbols dictate Th (big in size), Co (medium), and $\mathrm{C}$ atoms (small), respectively. (b) Measured temperature dependence data of magnetic susceptibility $\chi(T)$ are shown in ZFC and FC modes. Transverse field $\mu \mathrm{SR}$ asymmetry spectra are shown at (c) $T=0.1 \mathrm{~K}$, (d) $T=3.5 \mathrm{~K}$ for $H=10 \mathrm{mT}$, and (e) $T=0.4 \mathrm{~K}$, (f) $T=3.5 \mathrm{~K}$ for $H=30 \mathrm{mT}$. The solid lines are fit using an oscillatory function with a Gaussian relaxation, plus a nondecaying oscillation that originates from muons stopping in the silver sample holder. The effect of the flux line lattice can be seen in the top panel as the strong Gaussian decay envelope of the oscillatory function. Above $T_{c}$, the depolarization is reduced, which is due to the randomly oriented array of nuclear magnetic moments.

superconducting gap symmetry of $\mathrm{ThCoC}_{2}$ compound using transverse (TF) and zero fields (ZF) $\mu$ SR measurements. We find the evidence of USC with nodal line $d$ wave superconductivity as manifested from the TF $\mu \mathrm{SR}$. These measurements are further supported by the calculations of the pairing symmetry with spin-fluctuation exchange pairing potential as computed directly from the densityfunctional theory (DFT) based band structure calculations for $\mathrm{ThCoC}_{2}$.

A polycrystalline sample of $\mathrm{ThCoC}_{2}$ was prepared by an arc melting of the stoichiometric amount of Th $(3 \mathrm{~N})$, Co $(4 \mathrm{~N})$, and $\mathrm{C}(5 \mathrm{~N})$ on a water-cooled $\mathrm{Cu}$ hearth in a highpurity Ar atmosphere [25]. The ingots were sealed in an evacuated quartz tube and annealed at $1100{ }^{\circ} \mathrm{C}$ for $336 \mathrm{hr}$ to get better sample quality. X-ray powder diffraction data revealed that $\mathrm{ThCoC}_{2}$ is a single phase with space group Amm2 [25]. The magnetization data were obtained using a quantum design superconducting quantum interference device. The $\mu \mathrm{SR}$ experiments were carried out on the MUSR spectrometer at the ISIS Pulsed Neutron and Muon Source of Rutherford Appleton Laboratory, U.K [37]. The $\mu \mathrm{SR}$ experiments were conducted in $\mathrm{ZF}$ and $\mathrm{TF}$ modes. Spin-polarized muons $(100 \%)$ were implanted into the sample, and the positrons from the resulting muon decay were collected in the detector positions either backward or forward of the initial muon-spin direction for the $\mathrm{ZF}$ geometry. For TF measurements, the spectrometer was rotated by $90^{\circ}$. The powdered sample of $\mathrm{ThCoC}_{2}$ was mounted on a silver holder $(99.995 \%)$, and the sample holder was placed in a dilution refrigerator that operated in the temperature range $0.1 \mathrm{~K} \leq T \leq 3.5 \mathrm{~K}$. Using three sets of orthogonal coils and an active compensation system, the stray magnetic fields at the sample position due to the Earth and neighboring instruments are canceled to within $1 \mu \mathrm{T}$. $\mathrm{TF}-\mu \mathrm{SR}$ experiments were performed in the superconducting mixed state in several applied magnetic fields between
10 and $50 \mathrm{mT}$, due to the low value of $\mu_{0} H_{c 2}(\sim 3 \mathrm{kOe})$. Data were obtained in the field-cooled mode where the magnetic field was applied above $T_{c}$ and the sample was then cooled down to the lowest temperature. The $\mu \mathrm{SR}$ data were analyzed using WIMDA software [38].

The superconducting transition temperature $T_{c}=2.3 \mathrm{~K}$ was confirmed by the magnetic susceptibility $\chi(T)$, as shown in Fig. 1(b). The observed superconducting volume obtained from $\chi(T)$ data was $100 \%$ of perfect diamagnetism. The flux line lattice (FLL) in the vortex state of a typeII superconductor leads to a distinctive field distribution in the sample, which can be detected in the $\mu \mathrm{SR}$ relaxation rate. Figures $1(\mathrm{c})-1(\mathrm{f})$ show the $\mathrm{TF}-\mu \mathrm{SR}$ asymmetry-time spectra above and below $T_{c}$ with applied field of $10 \mathrm{mT}$ and $30 \mathrm{mT}$. Below $T_{c}$, the TF- $\mu \mathrm{SR}$ precession signal decays with time due to the distribution of fields associated with the FLL. The analysis of $\mathrm{TF}-\mu \mathrm{SR}$ asymmetry spectra was carried out in the time domain using a sinusoidal function damped with Gaussian relaxation plus a nondecaying oscillation that originates from muons stopping in the silver sample holder, $G_{z}(t)=A_{1} \cos \left(2 \pi \nu_{1} t+\Phi\right) \exp \left[-\left(\sigma^{2} t^{2} / 2\right)\right]+$ $A_{2} \cos \left(2 \pi \nu_{2} t+\Phi\right)$, where $A_{1}$ and $A_{2}$ are the sample and background asymmetries, $\nu_{1}$ and $\nu_{2}$ are the frequencies of the muon precession signal from the sample and background signal, respectively, with a phase angle $\Phi$ and where $\gamma_{\mu} / 2 \pi=135.5 \mathrm{MHz} \mathrm{T}^{-1}$ is the muon gyromagnetic ratio. $\sigma$ is the overall muon depolarization rate. It contains contributions from both the vortex lattice $\left(\sigma_{s c}\right)$ and nuclear dipole moments $\left(\sigma_{n m}\right.$, which is assumed to be temperature independent) [where $\left.\sigma=\sqrt{\left(\sigma_{s c}^{2}+\sigma_{n m}^{2}\right)}\right] \cdot \sigma_{s c}$ was determined by quadratically deducting the background nuclear dipolar depolarization rate obtained from spectra measured above $T_{c}$, which is shown in Fig. 2(a).

As can be seen in Fig. 2(a), $\sigma_{s c}$ depends on the applied field. Isothermal cuts perpendicular to the temperature 

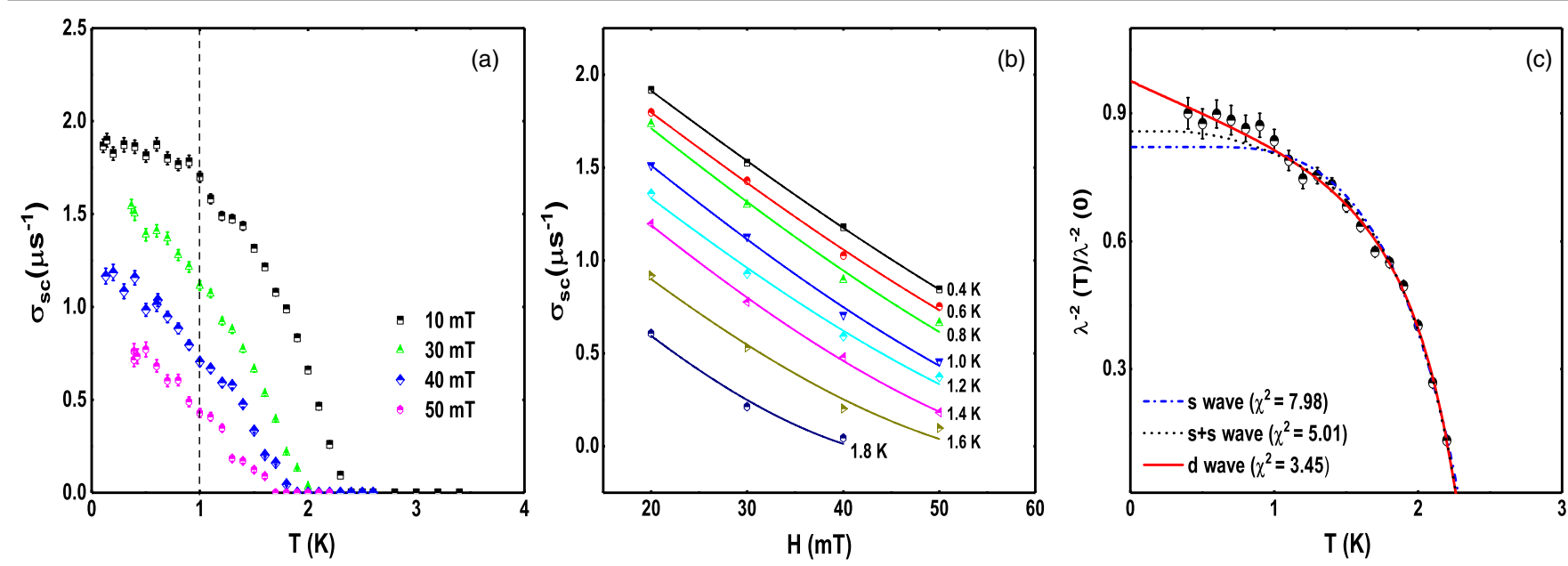

FIG. 2. (a) Temperature dependence of the TF- $\mu \mathrm{SR}$ spin-depolarization rate collected in a range of fields $10 \mathrm{mT} \leq H \leq 50 \mathrm{mT}$. The dashed line shows an example of the isothermal cuts used to find the field dependence of $\sigma_{s c}$ at a particular temperature. (b) Field dependence of the muon spin-depolarization rate is shown for a range of different temperatures. The solid lines are the results of fitting the data using the Brandt equation (Ref. [39]) as discussed in the text. (c) Temperature dependence of the inverse magnetic penetration depth squared is shown here. The lines show the fits using $s$ wave (dashed), $s+s$ wave (dotted), and $d$ wave (solid) gap functions.

axis of the $\sigma_{s c}$ data sets were used to determine the $H$ dependence of the depolarization rate $\sigma_{s c}(H)$ displayed in Fig. 2(b). In case of $\mathrm{ThCoC}_{2}$, the value of $H_{c 2}$ is smaller, so the superconducting $\sigma_{s c}$ is field dependent. Brandt [39] suggested a useful relation that describe the field dependence for a hexagonal lattice, which is valid over the field range examined in this experiment, $\sigma_{s}\left[\mu \mathrm{s}^{-1}\right]=4.854 \times 10^{4}\left(1-H_{\mathrm{ext}} / H_{c 2}\right) \times\{1+1.21[1-$ $\left.\left.\sqrt{\left(H_{\text {ext }} / H_{c 2}\right)}\right]^{3}\right\} \lambda(T)^{-2} \mathrm{~nm}^{-2}$, to estimate the temperature dependence of the inverse-squared penetration depth, $\lambda^{-2}$ and $\mu_{0} H_{c 2}$ (this equation is valid for $H_{\text {ext }} / H_{c 2}<0.25$ ). The resulting fits to the $\sigma_{s c}(H)$ data are displayed as solid lines in Fig. 2(b). The temperature dependence of $\lambda^{-2}$ is presented in Fig. 2(c). We can model the temperature dependence of the $\lambda^{-2}$ by the following equation [40,41], $\left[\lambda^{-2}\left(T, \Delta_{0, i}\right) / \lambda^{-2}\left(0, \Delta_{0, i}\right)\right]=1+(1 / \pi) \int_{0}^{2 \pi} \int_{\Delta(T)}^{\infty}(\delta f / \delta E) \times$ $\left.\left(E d E d \phi / \sqrt{E^{2}-\Delta(T}\right)^{2}\right)$, where $f=\left[1+\exp \left(-E / k_{B} T\right)\right]^{-1}$ is the Fermi function. The gap is given by $\Delta_{i}(T, 0)=$ $\Delta_{0, i} \delta\left(T / T_{c}\right) g(\phi)$, whereas $g(\phi)$ refers to the angular dependence of the superconducting gap function, and $\phi$ is the azimuthal angle along the Fermi surface. The $T$ dependence of the superconducting gap is approximated by the relation $\delta\left(T / T_{c}\right)=\tanh \left\{1.82\left[1.018\left(T_{c} / T-1\right)\right]^{0.51}\right\}$. $g(\phi)$ is substituted by (1) one for a single gap $s$ or a two-gap $s+s$ wave pairing, and (ii) $|\cos (2 \phi)|$ for a $d$-wave gap with line nodes.

We have analyzed the temperature dependence of $\lambda^{-2}$ based on three different models, a single-gap isotropic $s$ wave, a line node $d$ wave, and two gap $(s+s)$ wave models. The results of the fits using these gap structures are shown by different lines (dashed, dotted, and solid) in Fig. 2(c). It is clear from Fig. 2(c) that the $s$ or $s+s$ wave models do not fit the data, and gave a larger value of $\chi^{2}=7.98(3)$ for the $s$ wave and $\chi^{2}=5.01$ (1) for the $s+s$ wave model. The $s$ or $s+s$ wave model fits give the BCS ratio to be $2 \Delta(0) / k_{B} T_{c}=5.32$ and $5.22 / 0.74$, respectively. As the square root field dependence of the electronic heat capacity suggests a presence of nodes in the superconducting gap symmetry, we therefore fit our data using a $d$ wave model with line nodes in the gap structure. The $d$ wave model gives the best description of the temperature dependence of $\lambda^{-2}$ of $\mathrm{ThCoC}_{2}$. The $\chi^{2}$ value reduced significantly to $3.45(1)$ for the $d$ wave case. It gives $\Delta(0)=$ $0.77(1) \mathrm{meV}$ and $T_{c}=2.3(1) \mathrm{K}$, with the BCS ratio of $2 \Delta(0) / k_{B} T_{c}=7.8$, which is significantly larger than the weak coupling BCS value of 3.53, indicating a strong coupling superconductivity in $\mathrm{ThCoC}_{2}$. Enhanced value of the ratio is also observed in other NCS superconductors such as $\operatorname{Re}_{6} \operatorname{Zr}$ [42] with $2 \Delta(0) / k_{B} T_{c}=4.2, \mathrm{BiPd}$ [43] with $2 \Delta(0) / k_{B} T_{c}=3.8, \mathrm{La}_{7} \operatorname{Ir}_{3}$ [44] with $2 \Delta(0) / k_{B} T_{c}=3.81$, $\mathrm{K}_{2} \mathrm{Cr}_{3} \mathrm{As}_{3}$ [45] with $2 \Delta(0) / k_{B} T_{c}=6.4$, and $\mathrm{Cs}_{2} \mathrm{Cr}_{3} \mathrm{As}_{3}$ [46] with $2 \Delta(0) / k_{B} T_{c}=6.0$. Further larger values of this ratio are also observed in many Fe-based USC and HTSC cuprates $[47,48]$. Our ZF- $\mu$ SR study on $\mathrm{ThCoC}_{2}$ shows a similar relaxation rate above and below $T_{c}$, indicting that time reversal symmetry is preserved, see details in Ref. [49].

Now we discuss our theoretical calculations. For the first-principles electronic structure calculation, we use the Vienna $a b$ initio simulation package (VASP) [50] and the Perdew-Burke-Ernzerhof (PBE) form for the exchangecorrelation functional [51]. To deal with the strong correlation effect of the $d$ electrons of the Th and Co atoms, we employed LDA $+U$ method with $U=5 \mathrm{eV}$ on both atoms (the $U$-dependent band structure, shown in Ref. [49], does not show any considerable change in the Fermi surface topology). We have recalculated the band structure with spin-orbit coupling, and no considerable change is obtained 


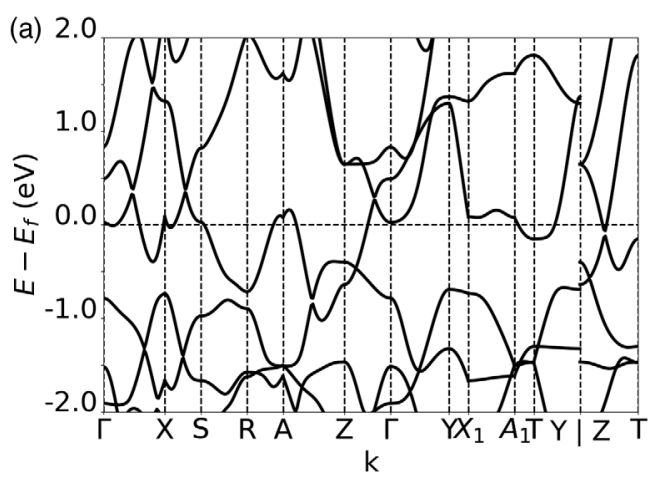

(b)

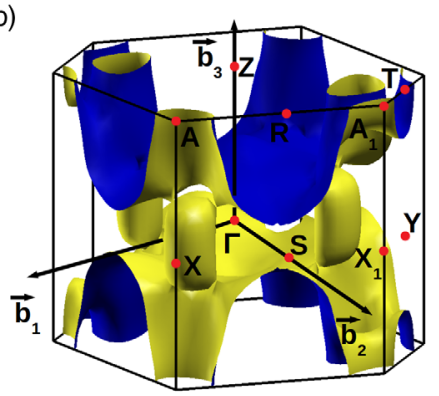

(c)

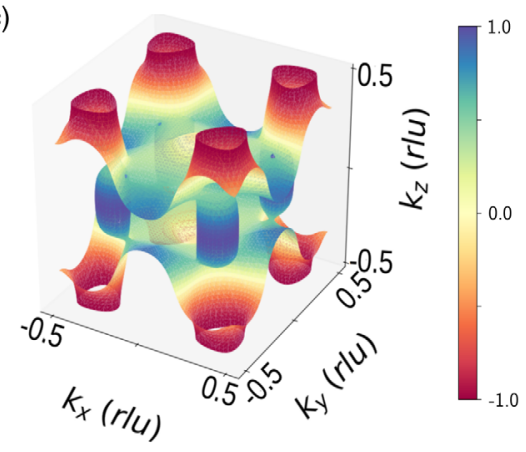

FIG. 3. (a) Computed result of DFT band structure is plotted along the standard high-symmetric directions for the orthorhombic crystal structure of $\mathrm{ThCoC}_{2}$. The low energy part of the band structure is dominated by Co- $d$ orbitals. (b) Corresponding Fermi surface topology is plotted in the first Brillouin zone of the lattice. The color in this plot does not have any specific meaning. The red dots show the high-symmetry points on the Brillouin zone and $\mathbf{b}_{\mathbf{1}}, \mathbf{b}_{2}$, and $\mathbf{b}_{3}$ are the reciprocal lattice vectors. (c) Computed pairing eigenfunction plotted in a color map of red (negative) to yellow (nodes) to blue (positive) color. The result is overlaid on the corresponding FS. We immediately observe the presence of nodal lines on the $k_{z}= \pm \pi / 2$ planes (visualized by yellow contour).

in the low-energy bands of present interests, presumably because of the weak RSOC strength of the Co atom that dominates the low-energy spectrum. The computed band structure is shown in Fig. 3(a). We notice that only a single band osculates around the Fermi level. This band is dominated by a Co- $d$ orbital with hybridization with the C- $p$ orbitals (with significantly less contributions from the $\mathrm{C}-p$ states to the low-energy band structure) (see the corresponding partial density of states result shown in Ref. [49]). The corresponding Fermi surface (FS) is shown in Fig. 3(b). The three-dimensional FS topology is quite interesting in this system, exhibiting a hole-like pocket around the zone corner ( $A$ point), but an electron-like topology around the $T$ point. In addition, as we scan across the $k_{z}$ direction, we notice that the Fermi pocket near the $k_{z}= \pm \pi$ plane first closes at discrete Fermi points, and then reopens into another pocket around some non-high-symmetric $k$ point. Such an interesting FS topological change in the 3D Brillouin zone (BZ) within a single band model implies the presence of saddle points in the electronic structure around the Fermi level. Hence one obtains a large density of states near the Fermi level, which is desirable for superconductivity. In addition, due to multiple pockets features in the FS, one expects large FS nestings, and hence unconventional superconductivity.

We perform a pairing symmetry calculation using a single-band Hubbard model. By carrying out a perturbative expansion of the Hubbard interaction, we obtain an effective pairing potential in the singlet and triplet pairing channels [52,53]

$$
\begin{aligned}
& V_{\uparrow \downarrow}(\mathbf{q})=\frac{U}{2}\left[1+3 U \chi^{s}(\mathbf{q})\right]+\frac{U}{2}\left[1-U \chi^{c}(\mathbf{q})\right], \\
& V_{\uparrow \uparrow}(\mathbf{q})=\frac{U}{2}\left[1-U \chi^{s}(\mathbf{q})\right]+\frac{U}{2}\left[1-U \chi^{c}(\mathbf{q})\right] .
\end{aligned}
$$

Here $\chi^{s / c}(\mathbf{q})$ are the spin and charge susceptibilities, decoupled within the random-phase approximation (RPA), as $\chi^{s / c}(\mathbf{q})=\chi^{0}(\mathbf{q}) /\left[1 \mp U \chi^{0}(\mathbf{q})\right]$ with $\chi^{0}(\mathbf{q})$ being a bare static Lindhard susceptibility. For such a potential, the BCS gap equation (for details see the Supplemental Material [49]) becomes

$$
\Delta_{\mathbf{k}}=-\lambda \sum_{\mathbf{k}^{\prime}} V\left(\mathbf{k}-\mathbf{k}^{\prime}\right) \Delta_{\mathbf{k}^{\prime}}
$$

This is an eigenvalue equation with the pairing strength $\lambda$ denoting the eigenvalue. For $V>0$, a positive eigenvalue $\lambda$ can commence with the corresponding eigenfunction $\Delta_{\mathbf{k}}$, which changes $\operatorname{sign}$ as $\operatorname{sgn}\left[\Delta_{\mathbf{k}}\right]=-\operatorname{sgn}\left[\Delta_{\mathbf{k}^{\prime}}\right]$, promoted by strong peak(s) in $V$ at $\mathbf{Q}=\mathbf{k}-\mathbf{k}^{\prime}$. Looking into the origin of $V$ in Eq. (1), we notice that $V$ inherits strong peaks from those in $\chi^{s / c}$, which is directly linked to the FS nesting feature embedded in $\chi^{0}$. In this way, the pairing symmetry of a system is intricately linked to the FS nesting instability. This theory of spin-fluctuation-driven superconductivity consistently links between the observed pairing symmetry and FS topology in many different unconventional superconductors $[52,54,55]$.

For the present FS topology presented in Fig. 3(c), we find that the nesting is dominant between the two FS pockets centered at the $k_{z}=0$ and $k_{z}= \pm \pi$ planes.

Our computed RPA spin susceptibility yields a strong nesting at $\mathbf{Q} \sim\left(0, \frac{1}{2}, \frac{1}{3}\right)$ and its equivalent points [49]. This $3 \mathrm{D}$ nesting is ingrained within the above pairing potential $V$, and naturally dictates a dominant pairing channel that changes sign between these two $k_{z}$ planes. In fact, our exact evaluation of the pairing eigenvalue gives the dominant pairing channel to be a spin-singlet pairing with $d_{z}$ wave pairing channel $\Delta_{\mathbf{k}}=\cos \left(k_{z}\right)$ with an eigenvalue of $\lambda \sim 0.2$. The computed pairing eigenfunction is shown in Fig. 3(c) on the FS in a color plot. The computed pairing channel does not acquire any in-plane anisotropy, and 
hence produces a line node on the $k_{z}= \pm \pi / 2$ planes. The experimental data fitted with the same nodal-like pairing channel indeed gives a better fit compared to other pairing functions.

In summary, we have investigated the gap symmetry of $\mathrm{ThCoC}_{2}$ using transverse field $\mu \mathrm{SR}$. Our $\mu \mathrm{SR}$ analysis confirmed that a nodal line $d$ wave model fits better than a single or two gaps isotropic $s$ wave model to the observed temperature dependence of the penetration depth. This finding is in agreement with the field dependent heat capacity, $\gamma(H) \sim \sqrt{H}$. The finding of the nodal line gap is further supported by our theoretical calculations. The sign reversal of the superconducting pairing structure generates a magnetic resonance peak below $T_{c}$ at the same nesting wave vector $\mathbf{Q}$ [56], as seen in cuprates [57], pnictides [58], and heavy-fermion superconductors [59]. Therefore, we propose the existence of such a resonance mode in the SC state, which can be observed by inelastic neutron scattering measurements. This Letter paves the way for further studies of the large numbers of unexplored NCS compounds with the $\mathrm{CeNiC}_{2}$ type structure for the hunt of the USC.

We would like to thanks, Professor A. M. Strydom for interesting discussions. A. B. would like to acknowledge DST India, for an Inspire Faculty Research Grant No. (DST/INSPIRE/04/2015/000169), FRC of UJ, NRF of South Africa, and ISIS-STFC for funding support. D. T. A. and A. D. H. would like to thank CMPC-STFC, Grant No. CMPC-09108. K. P. would like to acknowledge DST India, for an Inspire Fellowship (IF170620). T. D. acknowledges financial support from the Science and Engineering Research Board (SERB) of the Department of Science \& Technology (DST), Government of India for the Start-Up Research Grant (Young Scientist), and also benefited from the financial support from the Infosys Science foundation under Young Investigator Award.

*amitava.bhattacharyya@rkmvu.ac.in †devashibhai.adroja@stfc.ac.uk tnmydas@gmail.com

[1] M. Smidman, M. B. Salamon, H. Q. Yuan, and D. F. Agterberg, Rep. Prog. Phys. 80, 036501 (2017).

[2] Non-Centrosymmetric Superconductors Introduction and Overview, edited by E. Bauer and M. Sigrist (Springer, New York, 2012).

[3] E. I. Rashba, Sov. Phys. Solid State 2, 1109 (1960).

[4] P. A. Frigeri, D. F. Agterberg, A. Koga, and M. Sigrist, Phys. Rev. Lett. 92, 097001 (2004).

[5] K. V. Samokhin, E. S. Zijlstra, and S. K. Bose, Phys. Rev. B 69, 094514 (2004); 70, 069902(E) (2004).

[6] S. J. Fujimoto, J. Phys. Soc. Jpn. 76, 051008 (2007).

[7] P. A. Frigeri, D. F. Agterberg, I. Milat, and M. Sigrist, arXiv: cond-mat/0505108.
[8] E. Bauer, G. Hilscher, H. Michor, C. Paul, E. W. Scheidt, A. Gribanov, Y. Seropegin, H. Noel, M. Sigrist, and P. Rogl, Phys. Rev. Lett. 92, 027003 (2004).

[9] M. Yogi, Y. Kitaoka, S. Hashimoto, T. Yasuda, R. Settai, T. D. Matsuda, Y. Haga, and Y. Ōnuki, P. Rogl, and E. Bauer, Phys. Rev. Lett. 93, 027003 (2004).

[10] I. Bonalde, W. Bramer-Escamilla, and E. Bauer, Phys. Rev. Lett. 94, 207002 (2005).

[11] N. Kimura, K. Ito, K. Saitoh, Y. Umeda, H. Aoki, and T. Terashima, Phys. Rev. Lett. 95, 247004 (2005).

[12] N. Kimura, Y. Muro, and H. Aoki, J. Phys. Soc. Jpn. 76, 051010 (2007).

[13] Y. Okuda, Y. Miyauchi, Y. Ida, Y. Takeda, C. Tonohiro, Y. Oduchi, T. Yamada, N. D. Dung, T. D. Matsuda, Y. Haga, T. Takeuchi, M. Hagiwara, K. Kindo, H. Harima, K. Sugiyama, R. Settai, and Y. Onuki, J. Phys. Soc. Jpn. 76, 044708 (2007).

[14] I. Sugitani, Y. Okuda, H. Shishido, T. Yamada, A. Thamizhavel, E. Yamamoto, T. D. Matsuda, Y. Haga, T. Takeuchi, R. Settai, and Y. Onuki, J. Phys. Soc. Jpn. 75, 043703 (2006).

[15] T. Kawai, H. Muranaka, M.-A. Measson, T. Shimoda, Y. Doi, T. Matsuda, Y. Haga, G. Knebel, G. Lapertot, D. Aoki, J. Flouquet, T. Takeuchi, R. Settai, and Y. Onuki, J. Phys. Soc. Jpn. 77, 064716 (2008).

[16] H. Wang, J. Guo, E. D. Bauer, V. A. Sidorov, H. Zhao, J. Zhang, Y. Zhou, Z. Wang, S. Cai, K. Yang, A. Li, X. Li, Y. Li, P. Sun, Y. feng Yang, Q. Wu, T. Xiang, J. D. Thompson, and L. Sun, Phys. Rev. B 97, 064514 (2018).

[17] F. Honda, I. Bonalde, K. Shimizu, S. Yoshiuchi, Y. Hirose, T. Nakamura, R. Settai, and Y. Onuki, Phys. Rev. B 81, 140507(R) (2010).

[18] H. Q. Yuan, D. F. Agterberg, N. Hayashi, P. Badica, D. Vandervelde, K. Togano, M. Sigrist, and M. B. Salamon, Phys. Rev. Lett. 97, 017006 (2006).

[19] H. Q. Yuan, M. B. Salamon, P. Badica, and K. Togano, Physica (Amsterdam) 403B, 1138 (2008).

[20] J. Chen, M. B. Salamon, S. Akutagawa, J. Akimitsu, J. Singleton, J. L. Zhang, L. Jiao, and H. Q. Yuan, Phys. Rev. B 83, 144529 (2011).

[21] E. Bauer, G. Rogl, X. Q. Chen, R. T. Khan, H. Michor, G. Hilscher, E. Royanian, K. Kumagai, D. Z. Li, Y. Y. Li, R. Podloucky, and P. Rogl, Phys. Rev. B 82, 064511 (2010).

[22] I. Bonalde, R. L. Ribeiro, and W. Brämer-Escamilla, G. Mu, and H. H. Wen, Phys. Rev. B 79, 052506 (2009).

[23] A. D. Hillier, J. Quintanilla, and R. Cywinski, Phys. Rev. Lett. 102, 117007 (2009).

[24] O. I. Bodak and E. P. Marusin, Dopov. Akad. Nauk Ukr. RSR, Ser. A 12, 1048 (1979).

[25] T. Grant, A. J. S. Machado, D. J. Kim, and Z. Fisk, Supercond. Sci. Technol. 27, 035004 (2014).

[26] T. W. Grant, O. V. Cigarroa, P. F. S. Rosa, A. J. S. Machado, and Z. Fisk, Phys. Rev. B 96, 014507 (2017).

[27] J. Chen, L. Jiao, J. L. Zhang, Y. Chen, L. Yang, M. Nicklas, F. Steglich, and H. Q. Yuan, New J. Phys. 15, 053005 (2013).

[28] D. A. Wright, J. P. Emerson, B. F. Woodfield, J. E. Gordon, R. A. Fisher, and N. E. Phillips, Phys. Rev. Lett. 82, 1550 (1999). 
[29] H. D. Yang and J. Y. Lin, J. Phys. Chem. Solids 62, 1861 (2001).

[30] H. P. van der Meulen, Z. Tarnawski, A. de Visser, J. J. M. Franse, J. A. A. J. Perenboom, D. Althof, and H. van Kempen, Phys. Rev. B 41, 9352 (1990).

[31] H. Takagi, R. J. Cava, H. Eisaki, J. O. Lee, K. Mizuhashi, B. Batlogg, S. Uchida, J. J. Krajewski, and W. F. Peck, Jr., Physica (Amsterdam) 228C, 389 (1994).

[32] S. V. Shulga, S.-L. Drechsler, G. Fuchs, K.-H. Müller, K. Winzer, M. Heinecke, and K. Krug, Phys. Rev. Lett. 80, 1730 (1998).

[33] Y. Takano, H. Takeya, H. Fujii, H. Kumakura, T. Hatano, and K. Togano, Appl. Phys. Lett. 78, 2914 (2001).

[34] D. C. Peets, G. Eguchi, M. Kriener, S. Harada, Sk. Md. Shamsuzzamen, Y. Inada, G.-Q. Zheng, and Y. Maeno, Phys. Rev. B 84, 054521 (2011).

[35] N. Kimura, K. Ito, H. Aoki, S. Uji, and T. Terashima, Phys. Rev. Lett. 98, 197001 (2007).

[36] J. Quintanilla, A. D. Hillier, J. F. Annett, and R. Cywinski, Phys. Rev. B 82, 174511 (2010).

[37] Dr. A. Bhattacharyya et al., Probing Non-Centrosymmetric Superconductors ThCoC2 with Muons (STFC ISIS Neutron and Muon Source, 2015), https://doi.org/10.5286/ISIS.E .61782541 .

[38] F. L. Pratt, Physica (Amsterdam) 289B-290B, 710 (2000).

[39] E. H. Brandt, Phys. Rev. B 68, 054506 (2003).

[40] A. Bhattacharyya, D. T. Adroja, A. D. Hillier, R. Jha, V. P. S. Awana, and A. M. Strydom, J. Phys. Condens. Matter 29, 265602 (2017).

[41] D. T. Adroja, A. Bhattacharyya, P. K. Biswas, M. Smidmann, A. D. Hillier, H. Mao, H. Luo, G. H. Cao, Z. Wang, and C. Wang, Phys. Rev. B 96, 144502 (2017).

[42] R. P. Singh, A. D. Hillier, B. Mazidian, J. Quintanilla, J. F. Annett, D. McK. Paul, G. Balakrishnan, and M. R. Lees, Phys. Rev. Lett. 112, 107002 (2014).

[43] Z. Sun, M. Enayat, A. Maldonado, C. Lithgow, E. Yelland, D. C. Peets, A. Yaresko, A. P. Schnyder, and P. Wahl, Nat. Commun. 6, 6633 (2015).

[44] J. A. T. Barker, D. Singh, A. Thamizhavel, A. D. Hillier, M. R. Lees, G. Balakrishnan, D. McK. Paul, and R. P. Singh, Phys. Rev. Lett. 115, 267001 (2015).

[45] D. T. Adroja, A. Bhattacharyya, M. Telling, Y. Feng, M. Smidman, B. Pan, J. Zhao, A. D. Hillier, F. L. Pratt, and A. M. Strydom, Phys. Rev. B 92, 134505 (2015).

[46] D. T. Adroja, A. Bhattacharyya, M. Smidman, A. D. Hillier, Y. Feng, B. Pan, J. Zhao, M. R. Lees, A. Strydom, and P. K. Biswas, J. Phys. Soc. Jpn. 86, 044710 (2017).
[47] A. Bhattacharyya, D. T. Adroja, M. Smidman, and V. K. Anand, Sci. China Phys. Mech. Astron. 61, 127402 (2018).

[48] M. Hashimoto, I. M. Vishik, R-H. He, T. P. Devereaux, and Z.-X. Shen, Nat. Phys. 10, 483 (2014).

[49] See Supplemental Material at http://link.aps.org/ supplemental/10.1103/PhysRevLett.122.147001 for more information on the calculational details, time reversal symmetry and zero field $\mu$ SR study.

[50] G. Kresse and J. Furthmuller, Phys. Rev. B 54, 11169 (1996).

[51] J. P. Perdew, K. Burke, and M. Ernzerhof, Phys. Rev. Lett. 77, 3865 (1996).

[52] D. J. Scalapino, E. Loh, Jr., and J. E. Hirsch, Phys. Rev. B 34, 8190(R) (1986); T. M. Rice and K. Ueda, Phys. Rev. B 34, 6420 (1986); J. R. Schrieffer, X. G. Wen, and S. C. Zhang, Phys. Rev. B 39, 11663 (1989); P. Monthoux, A. V. Balatsky, and D. Pines, Phys. Rev. Lett. 67, 3448 (1991); M. Sigrist and K. Ueda, Rev. Mod. Phys. 63, 239 (1991); D. J. Scalapino, Rev. Mod. Phys. 84, 1383 (2012).

[53] K. Kubo, Phys. Rev. B 75, 224509 (2007).

[54] P. J. Hirschfeld, M. M. Korshunov, and I. I. Mazin, Rep. Prog. Phys. 74, 124508 (2011); S. Graser, T. A. Maier, P. J. Hirschfeld, and D. J. Scalapino, New J. Phys. 11, 025016 (2009); T. Das and A. V. Balatsky, Phys. Rev. B 84, 014521 (2011); Z.-J. Yao, J.-X. Li, and Z. D. Wang, New J. Phys. 11, 025009 (2009).

[55] T. Das, J.-X. Zhu, and M. J. Graf, Sci. Rep. 5, 8632 (2015); H. Ikeda, M.-T. Suzuki, and R. Arita, Phys. Rev. Lett. 114, 147003 (2015).

[56] I. Eremin, D. K. Morr, A. V. Chubukov, K. H. Bennemann, and M. R. Norman, Phys. Rev. Lett. 94, 147001 (2005); T. Das, R. S. Markiewicz, and A. Bansil, Phys. Rev. B 85, 064510 (2012); T. A. Maier, S. Graser, D. J. Scalapino, and P. Hirschfeld, Phys. Rev. B 79, 134520 (2009); T. Das and A. V. Balatsky, Phys. Rev. Lett. 106, 157004 (2011); T. Nomoto and H. Ikeda, Phys. Rev. B 90, 125147 (2014).

[57] M. Fujita, H. Hiraka, M. Matsuda, M. Matsuura, J. M. Tranquada, S. Wakimoto, G. Xu, and K. Yamada, J. Phys. Soc. Jpn. 81, 011007 (2012).

[58] P. Dai, Rev. Mod. Phys. 87, 855 (2015).

[59] C. Stock, C. Broholm, Y. Zhao, F. Demmel, H. J. Kang, K. C. Rule, and C. Petrovic, Phys. Rev. Lett. 109, 167207 (2012); O. Stockert, J. Arndt, E. Faulhaber, C. Geibel, H. S. Jeevan, S. Kirchner, M. Loewenhaupt, K. Schmalzl, W. Schmidt, Q. Si, and F. Steglich, Nat. Phys. 7, 119 (2011). 


\title{
Evidence of a Nodal Line in the Superconducting Gap Symmetry of Noncentrosymmetric $\mathrm{ThCoC}_{2}$
}

\author{
A. Bhattacharyya,,${ }^{1,2,3, *}$ D. T. Adroja,${ }^{2,3, \dagger}$ K. Panda,${ }^{1}$ Surabhi Saha, ${ }^{4}$ Tanmoy Das,${ }^{4,}{ }^{\ddagger}$ A. J. \\ S. Machado, ${ }^{5}$ O. V. Cigarroa, ${ }^{5,6}$ T. W. Grant,${ }^{5,6}$ Z. Fisk, ${ }^{6}$ A. D. Hillier, ${ }^{2}$ and P. Manfrinetti ${ }^{7}$ \\ ${ }^{1}$ Department of Physics, Ramakrishna Mission Vivekananda Educational and Research Institute, \\ Belur Math, Howrah 711202, West Bengal, India \\ ${ }^{2}$ ISIS Facility, Rutherford Appleton Laboratory, Chilton, Didcot Oxon, OX11 0QX, United Kingdom \\ ${ }^{3}$ Highly Correlated Matter Research Group, Physics Department, \\ University of Johannesburg, PO Box 524, Auckland Park 2006, South Africa \\ ${ }^{4}$ Department of Physics, Indian Institute of Science, Bangalore 560012, India \\ ${ }^{5}$ Escola de Engenharia de Lorena, Universidade de São Paulo, \\ P.O.Box 116, Lorena, São Paulo, 12602-810, Brazil \\ ${ }^{6}$ Department of Physics and Astronomy, University of California-Irvine, Irvine, CA 92697, USA \\ ${ }^{7}$ Department of Chemistry, University of Genova, 16146 Genova, Italy
}

(Dated: April 4, 2019)

\section{TIME REVERSAL SYMMETRY}

The time evolution of the zero field $(\mathrm{ZF})-\mu \mathrm{SR}$ spectra of $\mathrm{ThCoC}_{2}$ is shown in Fig.S1 for $T=0.4 \mathrm{~K}$ and 3.5 $\mathrm{K}$. In this relaxation experiment, any muons stopped on the silver sample holder gave a time independent background. No signature of precession is visible (either at $0.4 \mathrm{~K}$ or $3.5 \mathrm{~K}$ ), ruling out the presence of a sufficiently large internal magnetic field as seen in magnetically ordered compounds. The only possibility is that the muon-spin relaxation is due to static, randomly oriented local fields associated with the electronic and nuclear moments at the muon site. The $\mathrm{ZF}-\mu \mathrm{SR}$ data are well described in terms of the damped Gaussian KuboToyabe (KT) function, $G_{z 2}(t)=A_{1} G_{K T}(t) e^{-\lambda t}+A_{b g}$ where $G_{K T}(t)=\left[\frac{1}{3}+\frac{2}{3}\left(1-\sigma_{K T}^{2} t^{2}\right) e^{\frac{-\sigma_{K T}^{2} t^{2}}{2}}\right]$ is Gaussian Kubo-Toyabe function, $\lambda$ is the electronic relaxation rate, $A_{1}$ is the initial asymmetry, $A_{b g}$ is the background. The parameters $\sigma_{K T}, A_{1}$, and $A_{b g}$ are found to be temperature independent. It is evident from the $\mathrm{ZF}-\mu \mathrm{SR}$ spectra that there is no noticeable change in the relaxation rates at $3.5 \mathrm{~K}\left(\geq T_{\mathbf{c}}\right)$ and $0.4 \mathrm{~K}\left(\leq T_{\mathbf{c}}\right)$. This indicates that the time-reversal symmetry is preserved upon entering the superconducting state. $\sigma_{K T}$ accounts for the Gaussian distribution of static fields from nuclear moments (the second moment of field distribution is $H_{\mu}^{2}$ $=\sigma^{2} / \gamma_{\mu}^{2}$, with muon gyromagnetic ratio $\gamma_{\mu}=135.53$ $\mathrm{MHz} / \mathrm{T})$. The fits of $\mu \mathrm{SR}$ spectra in Fig.S1 by the decay function gave $\sigma_{K T}=0.31(7) \mu \mathrm{s}^{-1}$ and $\lambda=0.12(6)$ $\mu \mathrm{s}^{-1}$ at $3.5 \mathrm{~K}$ and $\sigma_{K T}=0.30(7) \mu \mathrm{s}^{-1}$ and $\lambda=0.11(9)$ $\mu \mathrm{s}^{-1}$ at $0.4 \mathrm{~K}$. The fits are shown by the solid lines in Fig.S1. Since within the error bars both $\sigma_{K T}$ and $\lambda$ at $T<T_{\mathbf{c}}$ and $T>T_{\mathbf{c}}$ are similar, there is no evidence of time-reversal symmetry breaking in $\mathrm{ThCoC}_{2}$. This result also confirm the absence of any kind of magnetic impurity in our sample. In Table I, we have given the fitted parameters obtained from the fit to the $\lambda^{-2}(T)$ data of
$\mathrm{ThCoC}_{2}$ using different gap models.

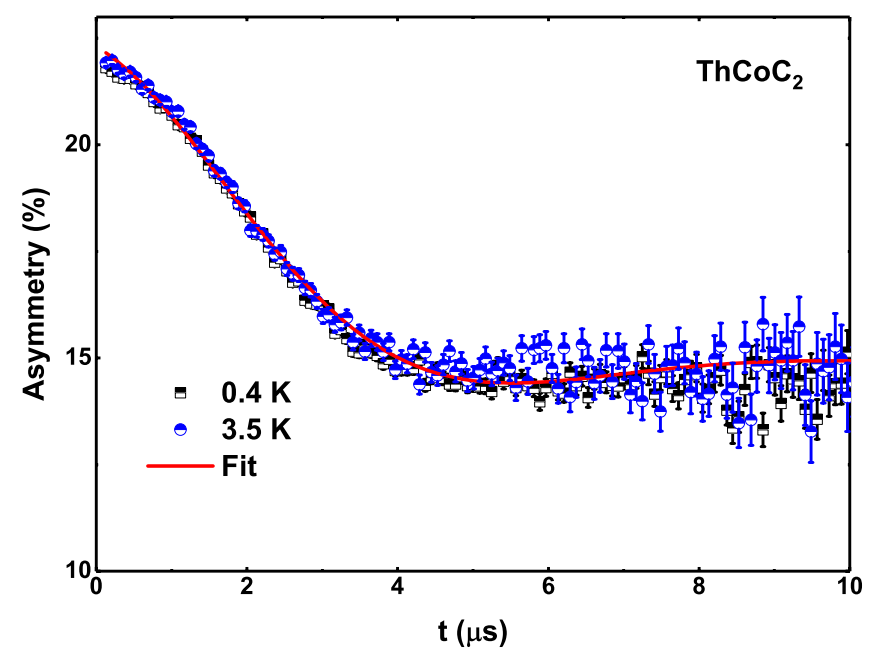

FIG. S1: (Color online) (a) Time dependence asymmetry spectra at $0.4 \mathrm{~K}$ and $3.5 \mathrm{~K}$ measured in zero magnetic field of $\mathrm{ThCoC}_{2}$ with $T_{\mathbf{c}}=2.3 \mathrm{~K}$. The lines are the results of the fits. The absence of extra relaxation below $T_{c}$ indicates no internal magnetic fields and, consequently, suggests that the superconducting state preserves time reversal symmetry.

TABLE I. Fitted parameters obtained from the fit to the $\lambda^{-2}(T)$ data of $\mathrm{ThCoC}_{2}$ using different gap models.

\begin{tabular}{lllll}
\hline \multirow{2}{*}{ Model } & $\mathrm{g}(\phi)$ & \multicolumn{4}{c}{ Gap Value } & Gap to $T_{\mathbf{c}}$ ratio & \multirow{2}{*}{$\chi^{2}$} \\
& & $\Delta(0)(\mathrm{meV})$ & $2 \Delta(0) / \mathrm{k}_{B} \mathrm{~T}_{C}$ & \\
\hline$d$-wave & $\cos (2 \phi)$ & 0.77 & 7.80 & 3.45 \\
$s+s$ wave & 1 & $0.52 ; 0.07$ & $5.22 ; 0.74$ & 5.01 \\
$s$-wave & 1 & 0.53 & 5.32 & 7.98 \\
\hline
\end{tabular}

\section{DFT CALCULATION}

For the first-principles electronic structure calculation, we used the Vienna ab initio Simulation Package 
(VASP) [1] and use the Perdew-Burke-Ernzerhof (PBE) form for the exchange-correlation functional [2]. The projector augmented wave (PAW) pseudo-potentials are used to describe the core electrons [3]. Electronic wavefunctions are expanded using plane waves up to cut-off energy of $500 \mathrm{eV}$. The Monkhorst-Pack k-mesh is set to $14 \times 14 \times 14$ in the Brillouin zone for the self-consistent calculation. All atoms are relaxed in each optimization cycle until atomic forces on each atom are smaller than $0.001 \mathrm{eV} \mathrm{nm}$. The lattice constants are obtained by relaxing the structure and with total energy minimization. Our obtained relaxed lattice parameters are $a=0.38493$ $\mathrm{nm}, b=0.38493 \mathrm{~nm}, c=0.39462 \mathrm{~nm}$, which are close to the experimental values $[4,5]$. To deal with the strong correlation effect of the $d$-electrons of the Th and Co atoms, we employed $\mathrm{LDA}+U$ method with $U=5 \mathrm{eV}$ on both atoms. We have recalculated the band structure with spin-orbit coupling, and no considerable change is obtained in the low-energy bands of present interests. We also did calculations for $U=3 \mathrm{eV}$ and $7 \mathrm{eV}$ to check the effect of $U$ on the band structure (see Fig. $2 \mathrm{~S}(\mathrm{c})$ )).

\section{DETAILS OF PAIRING EIGENVALUE CALCULATIONS}

Our calculation of pairing interaction and pairing eigenvalue originating from spin-fluctuation is done by directly including the DFT band structure in a threedimensional Brillouin zone (BZ). We start with a singleband Hubbard model, as dictated by the DFT calculation, which is given by

$$
H=\sum_{\mathbf{k}, \sigma=\uparrow, \downarrow} \xi_{\mathbf{k}} c_{\mathbf{k} \sigma}^{\dagger} c_{\mathbf{k} \sigma}+U \sum_{\mathbf{k}, \mathbf{k}^{\prime}, \mathbf{q}} c_{\mathbf{k} \uparrow}^{\dagger} c_{\mathbf{k}+\mathbf{q} \uparrow} c_{\mathbf{k}^{\prime} \downarrow}^{\dagger} c_{\mathbf{k}^{\prime}-\mathbf{q} \downarrow},
$$

where $c_{\mathbf{k} \sigma}^{\dagger}\left(c_{\mathbf{k} \sigma}\right)$ is the creation (annihilation) operator for non-interacting electron with momentum $\mathbf{k}$, and spin $\sigma=\uparrow / \downarrow$, and $\xi_{\mathbf{k}}$ is the corresponding band structure, taken directly from the DFT calculation. The second term is the Hubbard interaction, written in the band basis with the onsite potential $U$. Perturbative expansion of the Hubbard term in the spin-singlet and spin-triplet pairing channels yields,

$$
\begin{aligned}
H= & \sum_{\mathbf{k}, \sigma=\uparrow, \downarrow} \xi_{\mathbf{k}} c_{\mathbf{k} \sigma}^{\dagger} c_{\mathbf{k} \sigma} \\
& +\sum_{\mathbf{k} \mathbf{k}^{\prime}, \sigma \sigma^{\prime}} V_{\sigma \sigma^{\prime}}\left(\mathbf{k}-\mathbf{k}^{\prime}\right) c_{\mathbf{k} \sigma}^{\dagger} c_{-\mathbf{k} \sigma^{\prime}}^{\dagger} c_{-\mathbf{k}^{\prime} \sigma^{\prime}} c_{\mathbf{k}^{\prime} \sigma} .
\end{aligned}
$$

Here $V_{\sigma \sigma^{\prime}}$ refers to the spin singlet and triplet case when $\sigma=\mp \sigma$, respectively. The corresponding (static) pairing potentials are given in Eqs.(2-3) in the main text. The bare susceptibility is

$$
\chi^{0}(\mathbf{q}, \omega)=-\sum_{\mathbf{k}} \frac{f\left(\xi_{\mathbf{k}}\right)-f\left(\xi_{\mathbf{k}+\mathbf{q}}\right)}{\omega-\xi_{\mathbf{k}}+\xi_{\mathbf{k}+\mathbf{q}}+\iota \delta},
$$

where $f$ is the corresponding Fermi-function, and $\delta$ is an infinitesimal number. Since we are interested in the static limit of the pairing potential, the imaginary part of the above particle-hole correlator does not contribute and we obtain a real pairing potential $V$. In the RPA channel, the spin and charge channels are decoupled, and due to different denominators $1 \mp U \chi^{0}$, the spin channel is strongly enhanced while simultaneously charge channel is suppressed. The method is a weak to intermediate coupling approach and thus the value of $U$ is restricted by the non-interacting bandwidth. We use $U=400 \mathrm{meV}$ in our numerical calculation and the result is reproduced with different values of $U$. We note that this value of $U$ is defined in the band basis, while the $\mathrm{LDA}+U$ is for the orbitals. The $k$-dependent of the pairing eigenfunction is dictated by the anisotropy in $V(\mathbf{q})$ which is directly related to the bare FS nesting character, this result remains unaffected by a particular choice of $U$ in the weak to intermediate coupling range. With increasing $U$, the overall strength of $V$ mainly increases and thus the pairing eigenvalue $\lambda$ also increases. Since in this work, we are primarily interested in uncovering the pairing eigenfunction, the value of $U$ remains irrelevant in this coupling strength.

From interacting Hamiltonian, we define the SC gap equation as [6-9],

$$
\begin{aligned}
\Delta_{\mathbf{k}} & =-\sum_{\mathbf{k}^{\prime}} V_{\sigma \sigma^{\prime}}\left(\mathbf{k}-\mathbf{k}^{\prime}\right)\left\langle c_{-\mathbf{k}^{\prime} \sigma} c_{\mathbf{k}^{\prime} \sigma^{\prime}}\right\rangle, \\
& =-\sum_{\mathbf{k}^{\prime}} V_{\sigma \sigma^{\prime}}\left(\mathbf{k}-\mathbf{k}^{\prime}\right) \frac{\Delta_{\mathbf{k}^{\prime}}}{2 \xi_{\mathbf{k}^{\prime}}} \tanh \left(\frac{\xi_{\mathbf{k}^{\prime}}}{k_{B} T_{c}}\right)
\end{aligned}
$$

In the limit $T \rightarrow 0$ we have $\left\langle c_{-\mathbf{k} \sigma} c_{\mathbf{k} \sigma^{\prime}}\right\rangle \rightarrow \lambda \Delta_{\mathbf{k}}$, leading to an eigenvalue equation as

$$
\Delta_{\mathbf{k}}=-\lambda \sum_{\mathbf{k}^{\prime}} V_{\sigma \sigma^{\prime}}\left(\mathbf{k}-\mathbf{k}^{\prime}\right) \Delta_{\mathbf{k}^{\prime}} .
$$

We solve this equation separately for spin singlet $(\sigma=$ $\left.-\sigma^{\prime}\right)$, and spin triplet $\left(\sigma=\sigma^{\prime}\right)$ cases. Since we restrict ourselves to the static limit, the above equation is solved for only Fermi momenta $\mathbf{k}_{F}, \mathbf{k}_{F}^{\prime}$ in which $V_{\sigma \sigma^{\prime}}\left(\mathbf{k}_{F}-\mathbf{k}_{F}^{\prime}\right)$ becomes a $n \times n$ matrix with $n$ being the number of discrete Fermi momenta considered in a 3D BZ.

We had presented results for $U=5 \mathrm{eV}$ for both Th$d$ and Co- $d$ orbital. Now we have carried out the $U$ dependence of the band structure as shown in Fig. S2 in the supplementary material. For a large variation of $U$ from $3 \mathrm{eV}$ to $7 \mathrm{eV}$, we find a systematic shift of the overall band structure. This changes the size of the Fermi surface but does not produce any topological change in it. The nesting wave vector, which dictates the pairing symmetry in our model, is aligned between the two flat areas of the Fermi surface along the $\mathbf{k}_{z}$ direction. Therefore, the systematic changes in the Fermi surface may only change the quantitative value of the nesting wave vector, but 
(a)

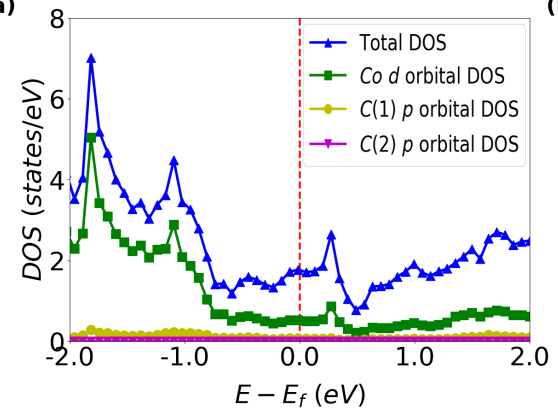

(b)

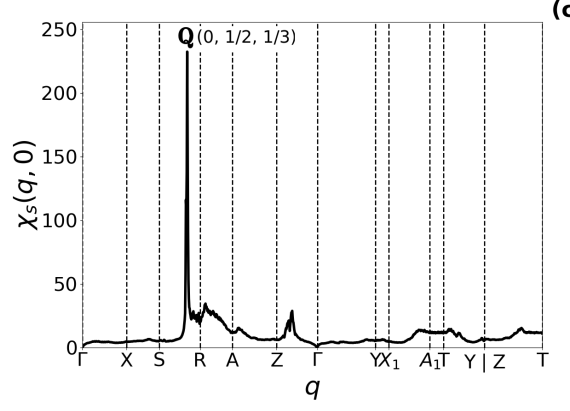

(c)

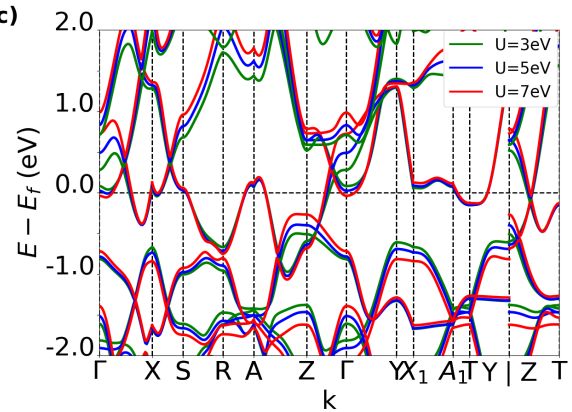

FIG. S2: (Color online) (a) Compute partial density of states (pDOS) plotted for three relevant orbitals near the Fermi level. We clearly observe that Co-d orbitals dominate the low-energy states. (b) The real part of the RPA susceptibility (static) in the spin-channel is plotted to the same high-symmetric directions. We observe a distinctly sharp peak at $\mathbf{Q}(0,1 / 2,1 / 3)$ in the susceptibility. This nesting peaks connect two Fermi surfaces across the $\mathbf{k}_{\mathbf{z}}=0$ plane. (c) Computed band structures for three different values of $U$. We notice a gradual shift of the bands, inducing a change in the FS area, but no change in the Fermi surface topology is observed in this wide range of $U . U=5$ (middle plot) is used in the paper.

qualitatively it remains the same. Therefore, the pairing eigenfunction also remains the same. In summary, for the single band Fermi surface topology of this system, the nesting driven pairing symmetry is a robust result to the practical range of $U$ used here.

* amitava.bhattacharyya@rkmvu.ac.in

$\dagger$ devashibhai.adroja@stfc.ac.uk

¥ tnmydas@gmail.com

[1] G. Kresse, and J. Furthmuller, Phys. Rev. B 54, 11169 (1996).

[2] J. P. Perdew, K. Burke, and M. Ernzerhof, Phys. Rev. Lett. 77, 3865 (1996).

[3] G. Kresse, and D. Joubert, Phys. Rev. B 59, 1758 (1999).

[4] M. H. Gerss and W. Jeitschko, Mat. Res. Bull., 21, 209216 (1986).

[5] T. Grant, A. J. S. Machado, D. J. Kim, and Z. Fisk, Su- percond. Sci. Technol. 27, 035004 (2014).

[6] D. J. Scalapino, E. Loh, Jr., and J. E. Hirsch, Phys. Rev. B 34, 8190 (R) (1986); T. M. Rice and K. Ueda, Phys. Rev. B 34, 6420 (1986); J. R. Schrieffer, X. G. Wen, and S. C. Zhang, Phys. Rev. B 39, 11663 (1989); P. Monthoux, A. V. Balatsky, and D. Pines, Phys. Rev. Lett. 67, 3448 (1991); Manfred Sigrist and Kazuo Ueda, Rev. Mod. Phys. 63, 239 (1991); D. J. Scalapino, Rev. Mod. Phys. 84, 1383 (2012).

[7] P. J. Hirschfeld, M. M. Korshunov, and I. I. Mazin, Rep. Prog. Phys. 74, 124508 (2011); S. Graser, T. A. Maier, P. J. Hirschfeld and D. J. Scalapino, New J. Phys. 11, 025016 (2009); T. Das and A. V. Balatsky, Phys. Rev. B 84, 014521 (2011); Zi-Jian Yao, Jian-Xin Li, and Z. D. Wang, New J. Phys., 11, 025009 (2009).

[8] T. Das, J.-X. Zhu and M. J. Graf, Sci. Rep. 5, 8632 (2015); H. Ikeda, M.-T. Suzuki and R. Arita, Phys. Rev. Lett. 114, 147003 (2015).

[9] Katsunori Kubo, Phys. Rev. B 75, 224509 (2007). 